\title{
Building interpretable models for polypharmacy prediction in older chronic patients based on drug prescription records
}

\author{
Simon Kocbek ${ }^{\text {Corresp., }}{ }^{1,2,3}$ ， Primoz Kocbek ${ }^{4}$, Andraz Stozer ${ }^{5}$, Tina Zupanic ${ }^{6}$, Tudor Groza ${ }^{7,8}$, Gregor Stiglic ${ }^{4,9}$ \\ ${ }^{1}$ Kinghorn Centre for Clinical Genomics, Garvan Institute of Medical Research, Sydney, NSW, Australia \\ 2 Advanced Analytics Institute, Faculty of Engineering and IT, University of Technology Sydney, Sydney, New South Wales, Australia \\ 3 Department of Computing and Information Systems, University of Melbourne, Melbourne, Victoria, Australia \\ 4 Faculty of Health Sciences, University Of Maribor, Maribor, Slovenia \\ 5 Institute of Physiology, Faculty of Medicine, University of Maribor, Maribor, Slovenia \\ 6 Healthcare Data Center, The National Institute of Public Health of the Republic of Slovenia, Ljubljana, Slovenia \\ 7 Kinghorn Centre for Clinical Genomics, Garvan Institute od Medical Research, Sydney, NSW, Australia \\ 8 St Vincent's Clinical School, Faculty of Medicine, UNSW Sydney, Sydney, NSW, Australia \\ 9 Faculty of Electrical Engineering and Computer Science, University of Maribor, Maribor, Slovenia \\ Corresponding Author: Simon Kocbek \\ Email address: skocbek@gmail.com
}

\section{Background}

Multimorbidity presents an increasingly common problem in older population, and is tightly related to polypharmacy, i.e. concurrent use of multiple medications by one individual. Detecting polypharmacy from drug prescription records is not only related to multimorbidity, but can also point at incorrect use of medicines. In this work, we build models for predicting polypharmacy from drug prescription records for newly diagnosed chronic patients. We evaluate the models' performance with a strong focus on interpretability of the results.

\section{Methods}

A centrally collected nationwide dataset of prescription records was used to perform electronic phenotyping of patients for the following two chronic conditions: type 2 diabetes mellitus (T2D) and cardiovascular disease (CVD). In addition, a hospital discharge dataset was linked to the prescription records. A regularized regression model was built for 11 different experimental scenarios on two datasets, and complexity of the model was controlled with a maximum number of dimensions (MND) parameter. Performance and interpretability of the model were evaluated with AUC, AUPRC, calibration plots, and interpretation by a medical doctor.

\section{Results}

For the CVD model, AUC and AUPRC values of $0.900(95 \% \mathrm{Cl}: 0.898-0.901)$ and $0.640(0.635-0.645)$ were reached, respectively, while for the T2D model the values were $0.808(0.803-0.812)$ and 0.732 (0.725 - 0.739). Reducing complexity of the model by $65 \%$ and $48 \%$ for CVD and T2D, resulted in $3 \%$ and $4 \%$ lower AUC, and 4\% and 5\% lower AUPRC values, respectively. Calibration plots for our models showed that we can achieve moderate calibration with reducing the models' complexity without significant loss of predictive performance.

\section{Discussion}


In this study, we found that it is possible to use drug prescription data to build a model for polypharmacy prediction in older population. In addition, the study showed that it is possible to find a balance between good performance and interpretability of the model, and achieve acceptable calibration at the same time. 


\title{
Building interpretable models for polypharmacy prediction in older chronic patients based on drug prescription records
}

Simon Kocbek ${ }^{1,2,3}$, Primoz Kocbek ${ }^{4}$, Andraz Stozer ${ }^{5}$, Tina Zupanic ${ }^{6}$, Tudor Groza ${ }^{1,7}$ and Gregor Stiglic ${ }^{4,8}$

${ }^{1}$ Kinghorn Center for Clinical Genomics, Garvan Institute of Medical Research, Sydney, Australia

${ }^{2}$ Advanced Analytics Institute, Faculty of Engineering and IT, University of Technology Sydney, Australia

${ }^{3}$ Dept of Computing and Information Systems, The University of Melbourne, Melbourne, Australia

${ }^{4}$ Faculty of Health Sciences, University of Maribor, Maribor, Slovenia

${ }^{5}$ Institute of Physiology, Faculty of Medicine, University of Maribor, Maribor, Slovenia

${ }^{6}$ Healthcare Data Center, The National Institute of Public Health of the Republic of Slovenia, Ljubljana, Slovenia

${ }^{7}$ St Vincent's Clinical School, Faculty of Medicine, UNSW Sydney, Sydney, Australia ${ }^{8}$ Faculty of Electrical Engineering and Computer Science, University of Maribor, Maribor, Slovenia

Corresponding Author:

Simon Kocbek ${ }^{1}$

Email address: skocbek@gmail.com

\begin{abstract}
Background

Multimorbidity presents an increasingly common problem in older population, and is tightly related to polypharmacy, i.e. concurrent use of multiple medications by one individual. Detecting polypharmacy from drug prescription records is not only related to multimorbidity, but can also point at incorrect use of medicines. In this work, we build models for predicting polypharmacy from drug prescription records for newly diagnosed chronic patients. We evaluate the models' performance with a strong focus on interpretability of the results.
\end{abstract}

\section{Methods}


A centrally collected nationwide dataset of prescription records was used to perform electronic phenotyping of patients for the following two chronic conditions: type 2 diabetes mellitus (T2D) and cardiovascular disease (CVD). In addition, a hospital discharge dataset was linked to the prescription records. A regularized regression model was built for 11 different experimental scenarios on two datasets, and complexity of the model was controlled with a maximum number of dimensions (MND) parameter. Performance and interpretability of the model were evaluated with AUC, AUPRC, calibration plots, and interpretation by a medical doctor.

\section{Results}

For the CVD model, AUC and AUPRC values of 0.900 (95\% CI: 0.898 - 0.901) and 0.640 (0.635 - 0.645) were reached, respectively, while for the T2D model the values were 0.808 (0.803 - 0.812) and $0.732(0.725-0.739)$. Reducing complexity of the model by $65 \%$ and $48 \%$ for CVD and T2D, resulted in 3\% and 4\% lower AUC, and 4\% and 5\% lower AUPRC values, respectively. Calibration plots for our models showed that we can achieve moderate calibration with reducing the models' complexity without significant loss of predictive performance.

\section{Discussion}

In this study, we found that it is possible to use drug prescription data to build a model for polypharmacy prediction in older population. In addition, the study showed that it is possible to find a balance between good performance and interpretability of the model, and achieve acceptable calibration at the same time. 


\section{Introduction}

Multimorbidity is becoming increasingly common, especially in older population. Despite the improvements in chronic disease treatment, the prevalence of multimorbid patients is still on the rise, although it is difficult to exactly define the multimorbidity [1]. However, it is known that prevalence of multimorbidity increases with age [2]. Polypharmacy or concurrent use of multiple medications by one individual is becoming another major health concern and is tightly related to multimorbidity. Especially in the older population, the number of concurrent health conditions is directly related to a number of medications prescribed, eventually resulting in polypharmacy [3].

Detecting polypharmacy from drug prescription records is not only related to multimorbidity, but can also point at incorrect use of medicines. According to estimates by the World Health Organisation (WHO) more than half of all medicines are prescribed, dispensed or sold inappropriately, and that half of all patients fail to take them correctly [4]. In the scope of the third global patient safety challenge, WHO addresses three areas of medication-related harm - i.e. highrisk situations, polypharmacy and transitions of care [5]. With the rapid introduction of the electronic health records (EHR), particularly at the primary healthcare level, it will be possible to effectively monitor and identify groups of patients or individuals at high risk for drug-induced or related health problems [6]. Additionally, linking different EHR repositories together [7] and solving challenges in capturing the data in electronic form [8] will allow further improvements of data driven techniques.

A great majority of studies on polypharmacy have focused on its potential negative consequences, e.g., nonadherence, interactions, and adverse drug reactions. Some researchers have also considered the effectiveness of interventions aimed at reducing polypharmacy, however, the factors and conditions leading to polypharmacy have received comparatively little attention. These factors can be broadly classified into four groups: (i) factors related to the health care system (e.g., life expectancy and novel therapies), (ii) factors related to patients (e.g., age and clinical conditions), (iii) factors related to physicians (e.g., guidelines and prescribing habits), and (iv) the interaction between patient and physician. In our study, we focused on medical therapy, more specifically on medications taken in the last three months in older patients with newly diagnosed chronic cardiovascular disease (CVD) type 2 diabetes mellitus (T2D) patients. Kumar et al. [9] demonstrate high prevalence of non-adherence problem as well as polypharmacy in patients with CVD and T2D where fears of drug toxicity are mentioned as a barrier to taking medicines.

Machine learning is becoming indispensable for solving problems in many disciplines, including healthcare. At the moment, we are witnessing the introduction of various machine learning approaches in different fields of healthcare that can help the professionals in improvement of diagnosis or prognosis and even displacing a lot of work done by radiologists and anatomical pathologists [10]. However, despite the ever-increasing prediction performance of the novel predictive modelling techniques, most of them still lack interpretability to offer actionable support 
41 for healthcare experts [11], [12]. Therefore, this study aims to offer more insight into balancing 42 the interpretability and predictive performance of the predictive models in healthcare. More 43 specifically, we evaluate different levels of interpretability offered by regularized logistic 44 regression modelling to predict polypharmacy based on prescription data in CVD and T2D 45 patients.

46

\section{Data and Methods}

48

\subsection{Study design and data source}

Two separate nationwide data sources were available for this study. The first dataset contained drug prescription records collected in Slovenia from 2008 to 2016, while the second dataset contained Slovenian hospital discharge records (primary healthcare level) from 2006 to 2016. Both datasets included patient identification information to allow linkage of data between years 2008 and 2016. All the data was collected centrally by National Institute of Public Health covering the whole population of Slovenia, which presents an important advantage compared to decentralised datasets where data linkage is not possible. The "Transparent reporting of a multivariable prediction model for individual prognosis or diagnosis" (TRIPOD) [13] was followed.

\subsection{Study setting}

60

A total of $94,475,895$ prescription entries for all patients who were prescribed at least one medication for T2D or CVD were obtained covering 755,966 unique patients (i.e., 402,286 males and 349,892 females, while 3,788 patients contained different genders at different time points and were later removed). The raw data contained 14 variables including anonymised patient id, patient gender, patient's geographical information, drug identifier, and the patient's doctor information. The hospital discharge data contained 1,740,610 entries covering 526,087 unique patients, who were prescribed at least one medication for T2D or CVD in the time period between 2006 and 2016. The discharge data was provided in two different formats, depending on time period when it was collected. Both formats included general information about the patient (e.g., age or anonymised identifier) and the admission (e.g., year and date of the hospitalisation or main diagnosis). International Classification of Diseases, revision 10 (ICD-10) was used to define specific diagnosis. ICD-encoding contains codes for diseases, signs and symptoms, abnormal findings, complaints, social circumstances, and external causes of injury or diseases. The main difference between the two hospital record formats was the number of ICD-10 codes. Data collected between 2006 and 2012 contained only primary and secondary diagnosis codes, while data collected between 2013 and 2016 contained primary and up to 19 additional diagnosis codes. To allow unbiased use of hospitalization data, only data from 2013 to 2016 was used in this study. 77

All records in both datasets were anonymised by the Slovenian National Institute of Public Health using the following three steps. First, a random identifier was assigned to each original patient 
81 provided, instead the patients were divided into age groups of 5 years. For example, the age group 820 would contain patients with ages from 0 to 4 years, the age group 1 would contain patients with 83 ages ranging from 5 to 9 years, etc. Finally, only the year and the month of the prescription were 84 given.

85

86

Anatomical Therapeutic Chemical Classification System (ATC) codes were used to code the 87 88 prescribed medications. ATC codes consist of up to 7 characters and provide the following information:

89 - L1: indicates the anatomical main group (one letter).

90 - L2: the therapeutic main group (two digits).

91 L3: the therapeutic/pharmacological subgroup (one character).

92 - L4: the chemical/therapeutic/pharmacological subgroup (one character).

93 - L5: the chemical substance (two digits).

94

95

96

97

98

99

100

101

102

103

104

105

106

107

108

109

110

111

112

113

114

115

116

117

118

119

120

\subsection{Preprocessing of the data}

First, we removed prescriptions for patients with different genders at different time points (manual inspection revealed an error in data) and prescriptions with no ATC codes (e.g., data entry errors or prescriptions of medical appliances). Next, for each ATC code, we also included the ATC3 code, i.e., a shorter L3 version of the full ATC code (e.g., for B01AA we would add B01).

Next, since the prevalence of polypharmacy increases by age, especially in older population aged 65+ [2], only patients born before 1960 (to consider the age group window) were included in the study. Since the dataset did not contain date of birth for patients, we used age groups to estimate dates of birth. For each patient, we calculated the maximum possible year of birth $(y b)$ from the patient's age group $(a g)$ and year $(y)$ of when the prescription was issued:

$y b(a g, d)=y-a g * 5$

Then we averaged all maximum possible years for each patient, rounded to the closest integer, and got the final approximate year of birth. The mean of year of birth for the 755,966 unique patients in our data was 1946.

Finally, we merged hospitalisation discharge data with prescription records.

\subsection{Electronic phenotyping}

Electronic phenotyping is often described as the process of identifying patients with a medical condition or characteristic [14]. In our work we had to identify patients with: a) polypharmacy, and b) newly diagnosed chronic CVD or T2D condition. We defined polypharmacy as a concurrent use of at least five medications. Concurrent use was defined as all medications that were prescribed in three consecutive months (e.g. January, February, March). 
121

122

123

124

125

126

127

128

129

130

131

132

133

134

135

136

137

138

139

140

141

142

143

144

145

146

147

148

149

150

151

152

153

154

155

156

157

158

As patients with a chronic condition $c$ we selected all those patients that were prescribed at least one medication for $c$ every three months, for a period of twelve months. Table 1 shows ATC codes for T2D and CVD. The latter were selected based on the recommendation by Huber et al. [15], which developed an updated and improved measure of patients' chronic disease.

As mentioned in Section 2.2, only data from 2013 until 2016 was used in the phenotyping stage. Figure 1 illustrates the electronic phenotyping steps. We selected January 2016 as the prediction time point (PTP) and filtered patients based on the chronic disease and polypharmacy conditions. For the former, we selected all non-chronic CVD and T2D that became chronic at PTP, and for the latter, we removed all patients with polypharmacy before PTP.

\subsection{Predictor and variables}

Final datasets contained 678 and 1,225 predictor variables for T2D and CVD respectively. The T2D dataset contained $44.9 \%$ of positive cases, while $21.8 \%$ of patients were positive in the CVD dataset. The latter indicates an unbalanced dataset which represents additional challenge for predictive techniques, and we had to consider this fact when evaluating our models. The predictor variables were arbitrary selected form a 3-months window before PTP and consisted of age, gender, hospitalisation, and ATC, ATC3 and ICD codes. Hospitalisation, ATC and ICD codes were indicator values $\{0,1\}$, Age was numeric, while Gender was a dichotomous variable. Table 2 presents statistics for predictive variables used in the prediction model for both datasets. Note that Age represents mean age in years (calculated from estimated year of birth), $n$ for Male, Female and Hosp represents number of instances, while $m$ for \#ATC, \#ATC3 and \#ICD represents number of variables with at least one positive instance.

\subsection{Predictive modeling}

Advanced statistical methods were applied to find patterns in the datasets for both chronic conditions, and a model to predict polypharmacy complications for patients was built. As one of our goals was to build interpretable models to increase usability, we restricted model building to regularized linear models, where model complexity (dimensionality) can be tuned. The latter also helps in avoiding overfitting, a problem in machine learning where models do not generalise well. We experimented with both L1-norm (LASSO) and broader elastic net regularization, however, the latter resulted in more complex modes with no performance gain, therefore we report results only for LASSO. The generalized linear model via penalized maximum likelihood LASSO regularization was used as defined by Friedman et al. [16]:

$$
\min _{\beta_{0}, \beta \frac{1}{N}} \sum_{i=1}^{N} w_{i} l\left(y_{i}, \beta_{0}+\beta^{T} x_{i}\right)+\lambda\left\|x_{i}\right\|_{1}
$$


159

160

161

162

163

164

165

166

167

168

169

170

171

172

173

174

175

176

177

178

179

180

181

182

183

184

185

186

187

188

189

190

191

192

193

194

195

196

197

198

where $i$ represents observations and it's negative log-likelihood contribution is noted as $l(y, n)$ with $w_{i}$ representing weights and tuning (shrinkage) parameter $\lambda$ controlling the overall strength of the penalty.

We further controlled the complexity of the model with the Maximal number of dimensions (MND) parameter with values from 10 to 100 in steps of 10 , where the $\lambda$ parameter was optimized with respect to the internal 5-fold cross validation. More precisely, in the MND models the last $\lambda$ value before the number of predictor variables reaches MND is selected.

\subsection{Model validation}

To evaluate our models, we focused on their predictive performance, which we describe in terms of discrimination and calibration. Discrimination measures the ability of a predictive model to separate outcomes, while calibration refers to the extent of the bias in the outcome of the model [17]. The discrimination can be measured by Area Under ROC Curve (AUC), i.e., the probability that the classifier will rank a randomly chosen positive case higher than a randomly chosen negative case. The ROC curve is plotted with Sensitivity or True Positive Rate (TPR) against the Fall-out or False Positive Rate (FPR), and AUC summarises the ROC curve into a single value by calculating the area of the convex shape below ROC. To obtain more details on predictive performance we also measured sensitivity, specificity, positive predictive value (PPV), negative predictive value (NPV), average number of selected features and percentage of positively predicted cases.

It is said that a model is "calibrated" when the predicted probability of a class matches the expected frequency of that class. Calibration can be visualized via a calibration plot, which plots class probabilities against those predicted by a single or multiple classifiers. In other words, calibration plots show observed proportion of events associated with a model's predicted risk, where the ideal calibration happens when both measures are equal. However, Van Calster et al. [18] defined a calibration hierarchy, with the lowest level 1 (mean calibration or "calibration-in-the-large") and highest level 4 (strong calibration), where they presented a strong case for using moderate or level 3 calibration (i.e., the average predicted risk is equal to the actual average risk), which can be assessed via calibration plot. Van Calster et al. also proved that moderate calibration guarantees that clinically harmless decisions are made based on the model. The calibration plots presented in this study show both level 1, where the ideal case is a 45-degree line with a slope coefficient 1 and intercept 0 [19], and level 3 calibration.

To validate the predictive models, we performed repeated cross-validation. More specifically, 10fold cross validation was used, whereby we randomly split data into 10 training/test sets for each model. We repeated this step 10 times, therefore we ended up with 100 experiments for each dataset. Instances in each fold were randomly selected. To evaluate and directly compare the models, the following two metrics were considered: AUC and Area Under the Precision Recall 
199 Curve (AUPRC). Similarly to AUC, AUPRC summarises the Positive Predictive Value (i.e, ratio 200 of correctly classified positive values to the number of all instances classified as positive) over 201 TPR curve into one number. AUPRC can often be more informative than AUC, especially for 202 unbalanced datasets [20], which was the case for the CVD dataset in this work.

203

204

Interpretability of models was measured by the number of selected variables in each experiment.

205

206

207

208

209

210

211

212

213

214

215

216

217

218

219

220

221

222

223

224

225

226

227

228

229

230

231

232

233

234

235

236

237 238 We reported the following results: a) number of all variables, and b) number of all variables that were selected in all repetitions of the experiments for different MND values (i.e., stable variables). In addition, a medical doctor manually inspected all selected variables for $\operatorname{MND}=\{10,20,50\}$ to evaluate the interpretability (i.e. extracted knowledge in form of variables from the logistic regression models) of the models from the medical point of view.

\section{Results}

This section presents the results in terms of predictive performance, calibration and selected variables for both CVD and T2D datasets. All results are reported for both datasets with different MND values to observe the influence of model complexity on performance.

\subsection{Predictive performance (AUC in AUPRC)}

Box plots in Figure 2 and Figure 3 illustrate AUC and AUPRC values for both chronic diseases and different MND values ranging from 10 to 100 and an additional model with no dimension reduction (NDR).

One can observe stabilisation of both performance metrics in both datasets immediately after the MND is increased from 10 to 20 . Even though a small increase in predictive performance can be observed when the complexity of the model increases, it is not significant, especially when MND increases to 50 and more selected variables.

More detailed predictive performance results including sensitivity, specificity, positive predictive value (PPV), negative predictive value (NPV), average number of selected features and percentage of positively predicted cases can be found in Supplement_T2D and Supplement_CVD.

\subsection{Calibration plots}

Figure 4 presents the calibration plots for both chronic conditions. Due to space limitations, we show calibration plots only for $\mathrm{MND}=\{10,20,50,100, \mathrm{NDR}\}$. The vertical axis of a calibration plot represents observed proportion of the class, while the horizontal axis represents the predicted probability.

Observing the calibration plots it can be noticed that the more complex models result in better calibration. However, the calibration improves significantly with the MND at 20 or higher. 
239

240

241

242

243

244

245

246

247

248

249

250

251

252

253

254

255

256

257

258

259

260

261

262

263

264

265

266

267

268

269

270

271

272

273

274

275

276

277

278

\subsection{Selected variables}

Due to space limitations, we list all selected variables for different experimental settings in Supplement_CVD and Supplement_T2D, while Table 3 (Table 4) summarises the number (ratio) of a) all variables, and b) variables that were selected in all repetitions of the experiments for different MND values (i.e., stable variables) and NDR. Please note, that the number of all selected variables in an experiment can be higher than the experiment's MND parameter, since we repeat each experiment 100 times and its selected variables do not necessarily always overlap (which is the case for stable variables).

\section{Discussion}

In this study, we found that it is possible to use drug prescription data to build a model for polypharmacy prediction. Results on Figure 2 and Figure 3 (details in Supplement_CVD and Supplement_T2D) show the maximum AUC (95\% CI) values of $0.900(0.898-0.901)$ and 0.808 (0.803 - 0.812) for CVD and T2D respectively, while AUPRC $(95 \% \mathrm{CI})$ reaches maximum values of $0.640(0.635-0.645)$ and $0.732(0.725-0.739)$ for CVD and T2D respectively.

We see that the difference between AUC and AUPRC was lower for T2D compared to CVD. The CVD dataset is skewed towards negative class and consists of $21,8 \%$ positive cases (compared to $44.9 \%$ positive cases in T2D). It was shown, that AUC can be misleading in terms of the reliability of classification performance in imbalanced datasets, whereas AUPRC can provide an accurate prediction of classification performance, since they evaluate true positive amongst positive predictions [20].

In addition, the study showed that it is feasible to find a balance between good performance and interpretability of the model. Figure 2 shows a slow decrease of the AUC performance for both datasets when complexity is controlled with MND. The difference with the maximum AUC values with dimensionality reduction is most notable with $\mathrm{MND}=10$, where $\mathrm{AUC}$ drops below 0.750 for both medical conditions. However, we can notice that increasing MND to 20, already improves performance significantly. Specifically, AUC of 0.875 (0.873 - 0.877) and 0.782 (0.777 - 0.787) is achieved for CVD and T2D respectively. The difference in AUC performance for MND=20 compared to NDR is only $3 \%$ and $4 \%$ for CVD and T2D respectively, while Table 4 shows that only $5 \%$ and $1 \%$ of all variables have been kept for CVD and T2D respectively $(35 \%$ and $52 \%$ of stable variables). The ratio between reduced complexity and decreased performance gets even smaller with higher MND values. For example, the maximum AUC value of 0.90 for CVD is achieved with $\mathrm{MND}=50$, with only $13 \%$ variables kept (67\% stable variables).

Similarly to AUC in Figure 2, results in Figure 3 show how AUPRC changes when we control MND. The lowest performance was obtained for $\mathrm{MND}=10$, while already with $\mathrm{MND}=20, \mathrm{AUPRC}$ values reach $96 \%$ (0.607) and 95\% (0.703) of the maximum AUPRC values for CVD and T2D, respectively. 
279

280

281

282

283

284

285

286

287

288

289

290

291

292

293

294

295

296

297

298

299

300

301

302

303

304

305

306

307

308

309

310

311

312

313

314

315

316

317

318

The AUC and AUPRC results show that while medical experts are able to work with much less complex models when reducing MND, this does not mean that they have to significantly sacrifice performance of the model.

The study also showed that it is possible to achieve acceptable calibration when reducing complexity of the model. Calibration plots presented in Figure 4 show the correlation between calibration and complexity of the model. Calster et al. [18] recommend that strong calibration should be desirable in cases of individualized decision support, but often stimulates too complex models and might even be counterproductive in other cases. Our results confirm this recommendation as the models with the best calibration result in the highest number of selected variables, but without significant improvement of predictive performance. Further recommendation by Calster et al. [18] introduces the so called moderate calibration defined by equality of the average predicted and the actual average risk. Moderate calibration can be observed in all models presented in our study.

Interpretation of selected variables by a medical doctor revealed that both CVD and T2D seem to be associated with polypharmacy independently of other medication [22], [23]. Moreover, in our models for both CVD and T2D, a large proportion of selected medications suggest other clinical conditions that were reported in a review of nine studies to be associated with polypharmacy, e.g., depression, asthma, and gout [3]. Further, some of the groups of drugs strongly suggest patient conditions other than well-defined diseases, such as declining nutrition and cognitive capacity that were also reported to be independently associated with polypharmacy [24]. Concerning particular groups of drugs as predictors of polypharmacy, studies show large variation and this is further complicated by the fact that study settings differ as well [21]. In a large study of an entire national population, Hovstadius et al. have found that the five most often prescribed drug groups in patients receiving polypharmacy were (listed in decreasing order occurrence) antibacterials, analgesics, psycholeptics, antithrombotic agents, and beta blocking agents [25]. In our study, analgesics and psycholeptics were included as features across all models for both CVD and T2D. Beta-blockers were excluded in the CVD group, but included in all of the models for T2D. Interestingly, in our case antibacterials were included only in the CVD model and the antithrombotic agents were robust predictive features for the T2D model, whereas they were included in the CVD model only above $\mathrm{MND}=50$.

Among the features most consistently selected in our models for both CVD and T2D were psycholeptics, psychoanaleptics, and antiepileptics. It should be noted that particularly the latter are sometimes used for indications other than epilepsy, e.g. mood stabilization. However, our finding corresponds with previous reports that people with mental health conditions and behavioral problems are at an increased risk for polypharmacy in general [26], [27] and that long-term use of some of the drugs from these groups carries the risk of metabolic dysregulation [28], falls [27] or 
even cognitive decline [29], which may precipitate a vicious cycle of receiving an increasing number of drugs.

Interestingly, compared with other studies reporting that women are more likely to receive polypharmacy [22], [30], [31] and that increasing age is a key determinant of polypharmacy exposure [21], [23], [3], [32]), in our sample, no robust association was found between polypharmacy and gender, and age was a feature selected only for the CVD model above $\mathrm{MND}=10$. It is possible that some of the features selected in models yielding better prediction might not reflect worsening physical health and thus a greater biological need for polypharmacy due to true multimorbidity, but may reflect patient transfer to an institution or change in residency, since it has been found that some of the medications that were robustly selected in our models are more frequently reported for patients in residential, as compared to community group homes or those living independently [26] and that nursing home residents are at an increased risk for polypharmacy [33].

The addition of diagnosis data (inpatient ICD codes) showed little improvement in our models, both in terms of gain in AUC or AUPRC and in terms of selected features in the models. Our explanation for this outcome is twofold; firstly there were only $13.3 \%$ hospitalization associated with CVD cases and even less $12.6 \%$ with T2D cases, which gives us a sparse matrix with little information gain. Secondly, drug prescriptions are usually to some degree associated with diagnoses of hospitalizations especially for elder chronic patients, which lowers the information gain for these features in our models even more. Secondly, drug prescriptions are usually to some degree associated with diagnoses of hospitalizations [34] especially for older chronic patients, which lowers the information gain for these features in our models even more.

The present study has some limitations that should be taken into the consideration. First, the data was restricted only to patients with at least one prescribed medication for either CVD or T2D. Second, since the records contain only month and year of the prescription or hospital admission, this influences our definitions for concurrent use of drugs, polypharmacy and chronic disease. Third, due to age groups, we were able to only estimate years of birth. Finally, a set of ATC codes for CVD was based on previous work of Huber et al. [15].

\section{Conclusion}

In this study we developed models to predict polypharmacy based on drug prescription and hospital discharge datasets. We focused on two common chronic conditions, i.e., CVD and T2D, since both are known to increase the risk of polypharmacy. Based on a centrally collected national prescription dataset, we defined and performed electronic phenotyping of chronic CVD and T2D patients with/and without polypharmacy. We also measured how increasing interpretability of predictive models by decreasing the number of variables included in the final model influences their performance. The interpretability of predictive models is important for the application of the 
359

360

361

362

363

364

365

366

367

368

369

370

371

372

373

374

375

376

377

378

379

380

381

382

383

384

385

386

387

388

389

390

391

392

393

394

395

396

397

398

399

400

401

402

403

proposed model in practice, especially in the context of learning healthcare systems where models are continuously adapted.

In the future, we plan to investigate performance of our models on other diseases and apply deep learning (DL) algorithms [35]. With DL, we expect less interpretable models with increased performance. We believe that our work has potential to positively influence drug prescription practices as discussed in [36].

\section{Reference}

[1] T. G. Willadsen, A. Bebe, R. Køster-Rasmussen, D. E. Jarbøl, A. D. Guassora, F. B. Waldorff, S. Reventlow, N. de F. Olivarius, "The role of diseases, risk factors and symptoms in the definition of multimorbidity - a systematic review," Scand. J. Prim. Health Care, vol. 34, no. 2, pp. 112-121, 2016.

[2] A. Calderón-Larrañaga, G. Santoni, H.X. Wang, A.K. Welmer, D. Rizzuto, D. L. Vetrano, A. Marengoni, L.. Fratiglioni , "Rapidly developing multimorbidity and disability in older adults: does social background matter?," J. Intern. Med., 2018.

[3] E. R. Hajjar, A. C. Cafiero, and J. T. Hanlon, "Polypharmacy in elderly patients," Am. J. Geriatr. Pharmacother., vol. 5, no. 4, pp. 345-351, 2007.

[4] Who, "The Pursuit of Responsible Use of Medicines: Sharing and Learning from Country Experiences," Vet. Rec., vol. 169, no. 9, p. 78, 2012.

[5] A. Sheikh, N. Dhingra-Kumar, E. Kelley, M. P. Kieny, and L. J. Donaldson, "The third global patient safety challenge: Tackling medication-related harm," Bulletin of the World Health Organization, vol. 95, no. 8, 2017.

[6] M. Molokhia and A. Majeed, "Current and future perspectives on the management of polypharmacy," BMC Family Practice, vol. 18, no. 1. 2017.

[7] S. Kocbek, L. Cavedon, D. Martinez, C. Bain, C. Mac. Manus, G. Haffari, I. Zukerman, K. Verspoor, "Text mining electronic hospital records to automatically classify admissions against disease: Measuring the impact of linking data sources," J. Biomed. Inform., vol. 64, pp. 158-167, 2016.

[8] G. Stiglic, P. Kocbek, N. Fijacko, A. Sheikh, and M. Pajnkihar, "Challenges associated with missing data in electronic health records: A case study of a risk prediction model for diabetes using data from Slovenian primary care," Health Informatics J., p. $146045821773328,2017$.

[9] K. Kanta, S. Greenfield, K. Raza, P. Gill, and R. Stack. "Understanding adherence-related beliefs about medicine amongst patients of South Asian origin with diabetes and cardiovascular disease patients: a qualitative synthesis," BMC endocrine disorders 16, no. 1 (2016): 24.

[10] Z. Obermeyer and E. J. Emanuel, "Predicting the Future - Big Data, Machine Learning, and Clinical Medicine," N. Engl. J. Med., vol. 375, no. 13, pp. 1216-1219, 2016.

[11] A. Holzinger, C. Biemann, C. S. Pattichis, D. B. Kell, "What do we need to build explainable AI systems for the medical domain?," arXiv preprint arXiv:1712.09923

[12] G. Stiglic, S. Kocbek, I. Pernek, P. Kokol., "Comprehensible predictive modeling using regularized logistic regression and comorbidity based features," PLoS One, vol. 10, no. $12,2015$. 
404 [13] G. S. Collins, J. B. Reitsma, D. G. Altman, and K. G. M. Moons, "Transparent reporting

405

406

407

408

409

410

411

412

413

414

415

416

417

418

419

420

421

422

423

424

425

426

427

428

429

430

431

432

433

434

435

436

437

438

439

440

441

442

443

444

445

446

447

448

449 of a multivariable prediction model for individual prognosis or diagnosis (TRIPOD): The TRIPOD Statement," European Urology, vol. 67, no. 6. pp. 1142-1151, 2015.

[14] J. M. Banda, Y. Halpern, D. Sontag, and N. H. Shah, "Electronic phenotyping with APHRODITE and the Observational Health Sciences and Informatics (OHDSI) data network.," AMIA Jt. Summits Transl. Sci. proceedings. AMIA Jt. Summits Transl. Sci., vol. 2017, pp. 48-57, 2017.

[15] C. A. Huber, T. D. Szucs, R. Rapold, and O. Reich, "Identifying patients with chronic conditions using pharmacy data in Switzerland: an updated mapping approach to the classification of medications," BMC Public Health, vol. 13, no. 1, p. 1030, 2013.

[16] J. Friedman, T. Hastie, and R. Tibshirani, "Regularization Paths for Generalized Linear Models via Coordinate Descent," J. Stat. Softw., vol. 33, no. 1, 2010.

[17] F. E. Harrell, K. L. Lee, and D. B. Mark, "Multivariable prognostic models: Issues in developing models, evaluating assumptions and adequacy, and measuring and reducing error," Stat. Med., vol. 15, no. 4, pp. 361-387, 1996.

[18] B. Van Calster, D. Nieboer, Y. Vergouwe, B. De Cock, M. J. Pencina, and E. W. Steyerberg, "A calibration hierarchy for risk models was defined: From utopia to empirical data," J. Clin. Epidemiol., vol. 74, pp. 167-176, 2016.

[19] E. W. Steyerberg, B. Van Calster, and M. J. Pencina, "Performance Measures for Prediction Models and Markers: Evaluation of Predictions and Classifications," Rev.

Española Cardiol. (English Ed., vol. 64, no. 9, pp. 788-794, 2011.

[20] T. Saito and M. Rehmsmeier, "The precision-recall plot is more informative than the ROC plot when evaluating binary classifiers on imbalanced datasets," PLoS One, vol. 10, no. 3, 2015.

[21] B. Hovstadius and G. Petersson, "Factors Leading to Excessive Polypharmacy," Clinics in Geriatric Medicine, vol. 28, no. 2. pp. 159-172, 2012.

[22] L. Bjerrum, J. Søgaard, J. Hallas, and J. Kragstrup, "Polypharmacy: Correlations with sex, age and drug regimen,” Eur. J. Clin. Pharmacol., vol. 54, no. 3, pp. 197-202, 1998.

[23] J. Jyrkkä, H. Enlund, M. J. Korhonen, R. Sulkava, and S. Hartikainen, "Patterns of drug use and factors associated with polypharmacy and excessive polypharmacy in elderly persons: Results of the kuopio 75 study: A cross-sectional analysis," Drugs and Aging, vol. 26, no. 6, pp. 493-503, 2009.

[24] J. Jyrkkä, H. Enlund, P. Lavikainen, R. Sulkava, and S. Hartikainen, “Association of polypharmacy with nutritional status, functional ability and cognitive capacity over a three-year period in an elderly population," Pharmacoepidemiol. Drug Saf., vol. 20, no. 5, pp. 514-522, 2011.

[25] B. Hovstadius, S. Tågerud, G. Petersson, and B. Åstrand, "Prevalence and therapeutic intensity of dispensed drug groups for individuals with multiple medications: A registerbased study of 2.2 million individuals," J. Pharm. Heal. Serv. Res., vol. 1, no. 4, pp. 145$155,2010$.

[26] M. O’Dwyer, J. Peklar, P. Mccallion, M. Mccarron, and M. C. Henman, "Factors associated with polypharmacy and excessive polypharmacy in older people with intellectual disability differ from the general population: A cross-sectional observational nationwide study," BMJ Open, vol. 6, no. 4, 2016.

[27] J. Peklar, M. Kos, M. O’Dwyer, M. McCarron, P. McCallion, R. A. Kenny, M. C. Henman, "Medication and supplement use in older people with and without intellectual disability: 
450

451

452

453

454

455

456

457

458

459

460

461

462

463

464

465

466

467

468

469

470

471

472

473

474

475

476

477

478

479

480

481

482

483

484

485

486

487

488

489

490

491

492

493

An observational, cross-sectional study," PLoS One, vol. 12, no. 9, p. e0184390, 2017.

[28] P. Gareri, P. De Fazio, S. De Fazio, N. Marigliano, G. F. Ibbadu, and G. De Sarro, "Adverse effects of atypical antipsychotics in the elderly: A review," Drugs and Aging, vol. 23, no. 12, pp. 937-956, 2006.

[29] R. Jenkins, "Use of psychotropic medication in people with a learning disability. [Review] [47 refs],” Br. J. Nurs., vol. 9, no. 13, pp. 844-50, 2000.

[30] S. I. Haider, K. Johnell, M. Thorslund, and J. Fastbom, "Analysis of the association between polypharmacy and socioeconomic position among elderly aged $\geq 77$ years in Sweden," Clin. Ther., vol. 30, no. 2, pp. 419-427, 2008.

[31] D. M. Qato, G. C. Alexander, R. M. Conti, M. Johnson, P. Schumm, and S. T. Lindau, "Use of prescription and over-the-counter medications and dietary supplements among older adults in the United States.,"JAMA, vol. 300, no. 24, pp. 2867-2878, 2008.

[32] R. B. Stewart and J. W. Cooper, "Polypharmacy in the Aged: Practical Solutions," Drugs \& Aging, vol. 4, no. 6. pp. 449-461, 1994.

[33] D. L. Vetrano, M. Tosato, G. Colloca, E. Topinkova, D. Fialova, J. Gindin, H. G. Van Der Roest, F. Landi, R. Liperoti, R. Bernabei, G. Onder, "Polypharmacy in nursing home residents with severe cognitive impairment: Results from the SHELTER Study," Alzheimer's Dement., vol. 9, no. 5, pp. 587-593, 2013.

[34] I. Klarin, A. Wimo, J. Fastbom, "The association of inappropriate drug use with hospitalisation and mortality," Drugs \& aging, 22(1), 69-82, 2005.R. Miotto, F. Wang, S.

[35] Wang, X. Jiang, and J. T. Dudley, "Deep learning for healthcare: review, opportunities and challenges," Brief. Bioinform., 2017.

[36] M. Molokhia, A, Majeed, "Current and future perspectives on the management of polypharmacy,” BMC Fam Pract, 18: 70, 2017.

\section{List of Figures}

Figure 1: Summary of filtering chronic and polypharmacy patients where two years of prescription data are considered. First, all data is partitioned into time periods of three consecutive months. Each three-month interval is used to: a) define the number of concurrent use of medications, and b) check for T2D or CVD medications. Next, the year before prediction time point (PTP) is used to remove all patients with previous polypharmacy (i.e., number of concurrent medications is higher than 4) or previous CVD or T2D chronic condition (i.e., at least one chronic medication is taken every 3 months). Finally, the year following PTP is used to select only the patients with a chronic condition, while polypharmacy in this year is used to define positive and negative patients.

Figure 2: Boxplots of CVD and T2D AUC values with 100 iterations at different MND values.

Figure 3: Boxplots of CVD and T2D AUPRC values with 100 iterations at different MND values.

Figure 4: Calibration plots for CVD $(A-E)$ and T2D $(F-J)$ for average probabilities and different MND values. Predicted probabilities from each fold were saved and averaged over 10 repetitions. For each calibration plot in the upper left corner the intercept value ("in the large") and slope is 
494 shown together with the AUC value or c-statistic. The main part of the plot is a flexible calibration 495 curve based on restricted cubic splines, with a pointwise $95 \%$ confidence interval (dashed lines), 496 followed by a case/non case histogram at the bottom.

497

498 List of Tables

499

500 Table 1: Selected ATC codes for CVD and T2D.

501

502 Table 2: Summary table for predictor variables.

503

504 Table 3: Number of all and stable selected variables in all experimental repetitions. Number of 505 stable variables is presented in brackets.

506

507 Table 4: Ratio of all and stable selected variables in all experimental repetitions. Ratio of stable 508 variables is presented in brackets. 


\section{Figure 1 (on next page)}

Summary of filtering chronic and polypharmacy patients where two years of prescription data are considered.

First, all data is partitioned into time periods of three consecutive months. Each three-month interval is used to: a) define the number of concurrent use of medications, and b) check for T2D or CVD medications. Next, the year before prediction time point (PTP) is used to remove all patients with previous polypharmacy (i.e., number of concurrent medications is higher than 4) or previous CVD or T2D chronic condition (i.e., at least one chronic medication is taken every 3 months). Finally, the year following PTP is used to select only the patients with a chronic condition, while polypharmacy in this year is used to define positive and negative patients. 


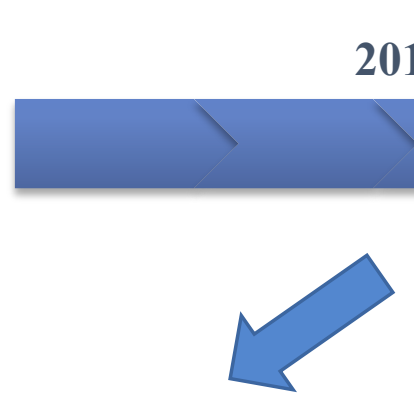

2015

$>\begin{aligned} & \text { Jan- } \\ & \text { Mar }\end{aligned}>\begin{aligned} & \text { Apr- } \\ & \text { Jun }\end{aligned}>$ Sul- $_{\text {Sept }}^{\text {Jul }}>\begin{aligned} & \text { Oct- } \\ & \text { Dec }\end{aligned}$

\section{Patients to keep}
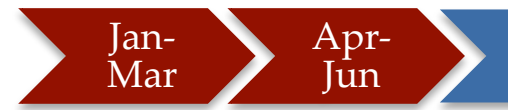
Jul-

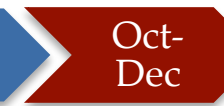

JanMar

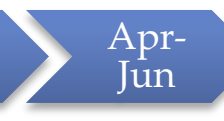

\section{$>\substack{\text { Iuli } \\ \text { Sept }}$}

\section{oat \\ Dec}

\section{Patients to remove}
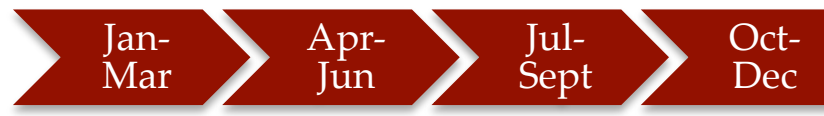

$>\operatorname{Jan}^{-}$
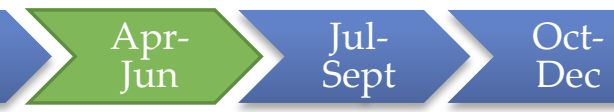

Peer] reviewing PDF | (2018:02:24836:1:1:NEW 11 Sep 2q18)
2016

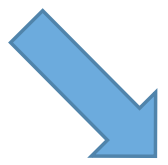

2016

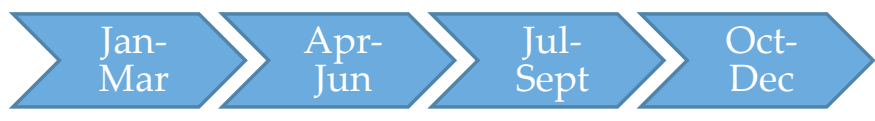

\section{Patients to keep}

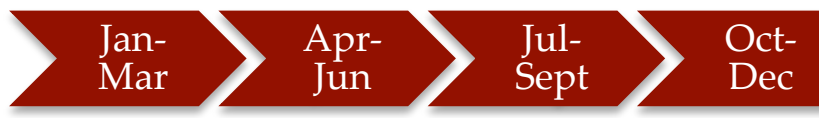

\section{Patients to remove}

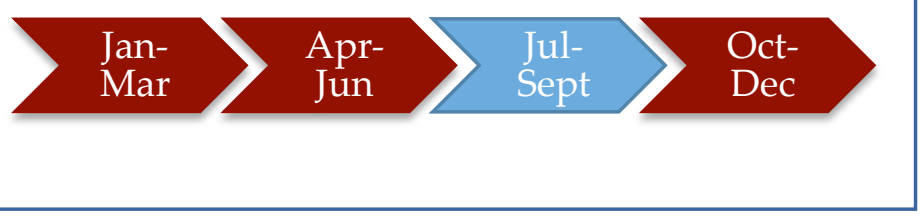


Figure 2 (on next page)

Boxplots of CVD and T2D AUC values with 100 iterations at different MND values. 
Manuscript to be reviewed

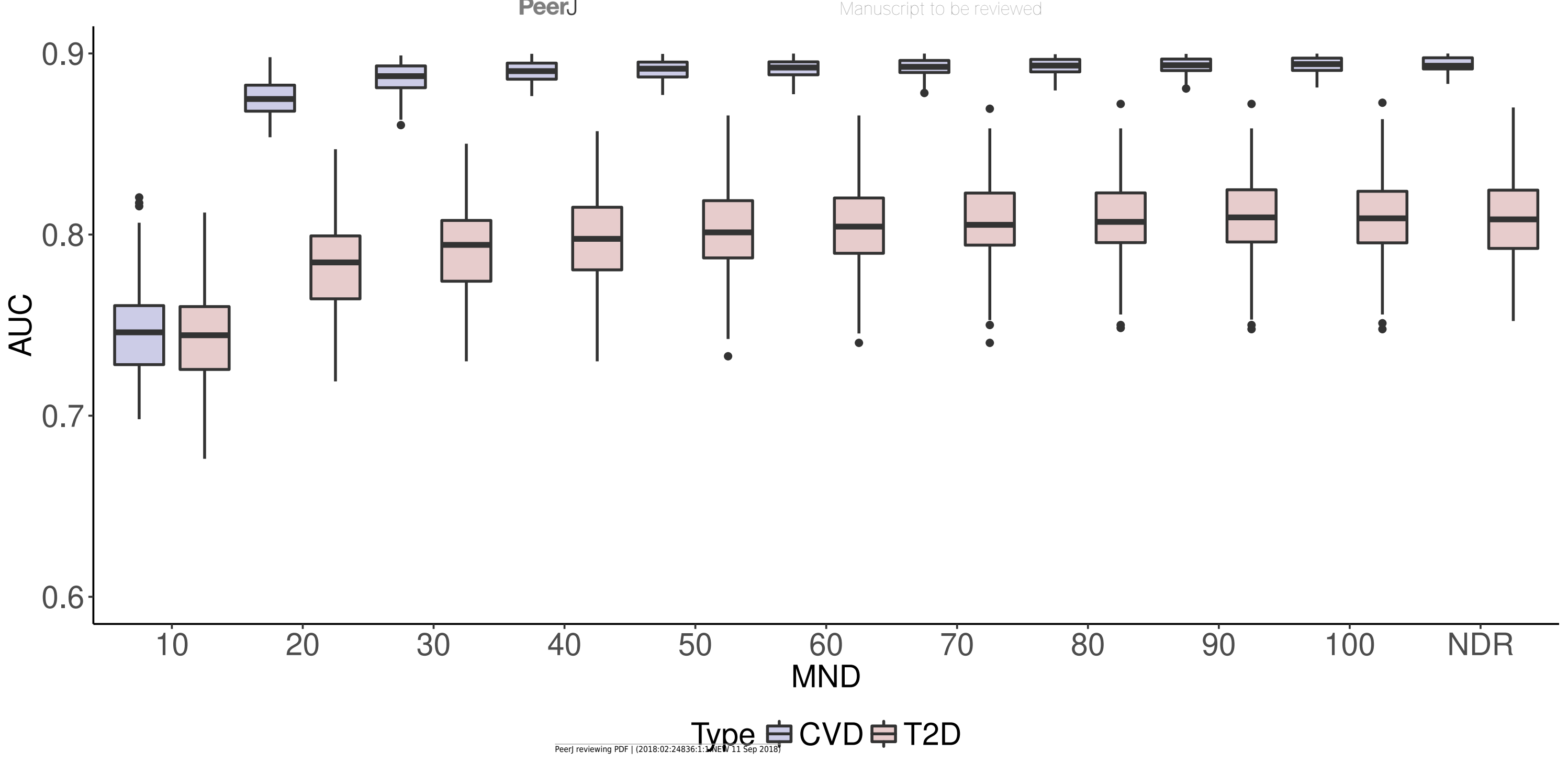


Figure 3 (on next page)

Boxplots of CVD and T2D AUPRC values with 100 iterations at different MND values. 
Figure 4 (on next page)

Calibration plots for CVD and T2D for average probabilities and different MND values.

Predicted probabilities from each fold were saved and averaged over 10 repetitions. For each calibration plot in the upper left corner the intercept value ("in the large") and slope is shown together with the AUC value or c-statistic. The main part of the plot is a flexible calibration curve based on loess, a k-nearest-neighbor-based meta-model combining regression models from nonparametric regression methods, with a pointwise $95 \%$ confidence interval, followed by a case/non case histogram at the bottom. 


\section{A}

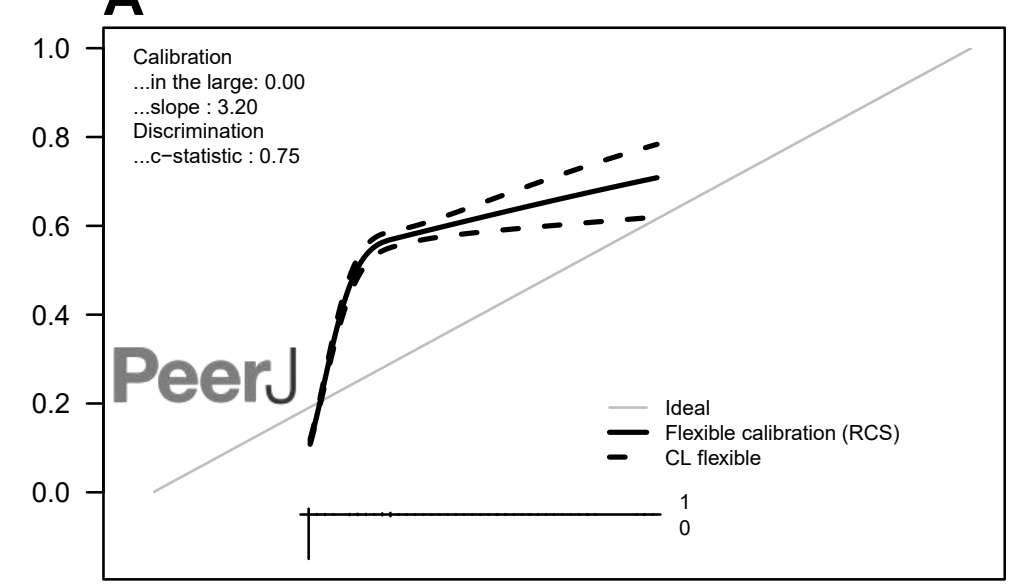

B

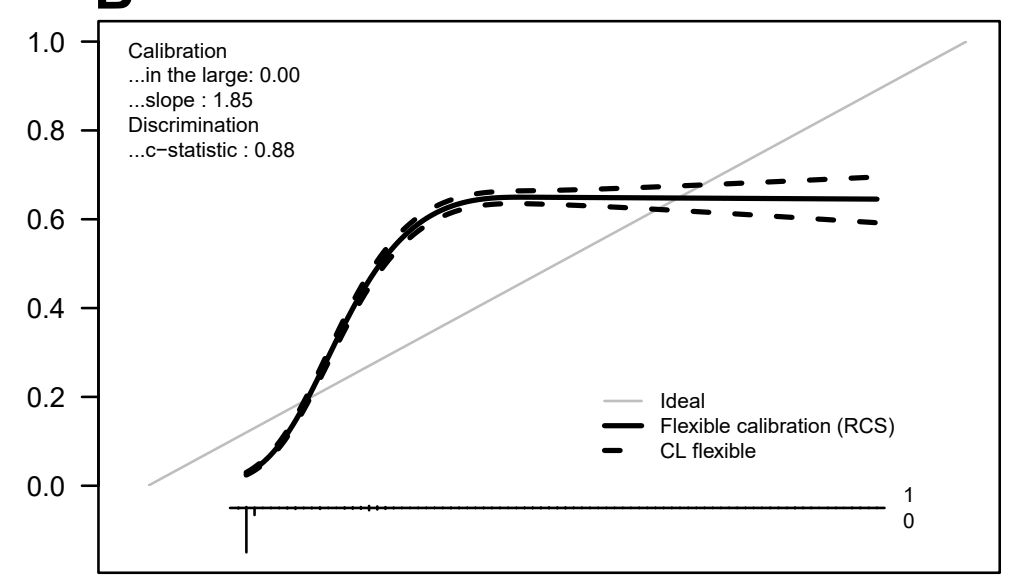

\section{C $\mathrm{MND}=50$}

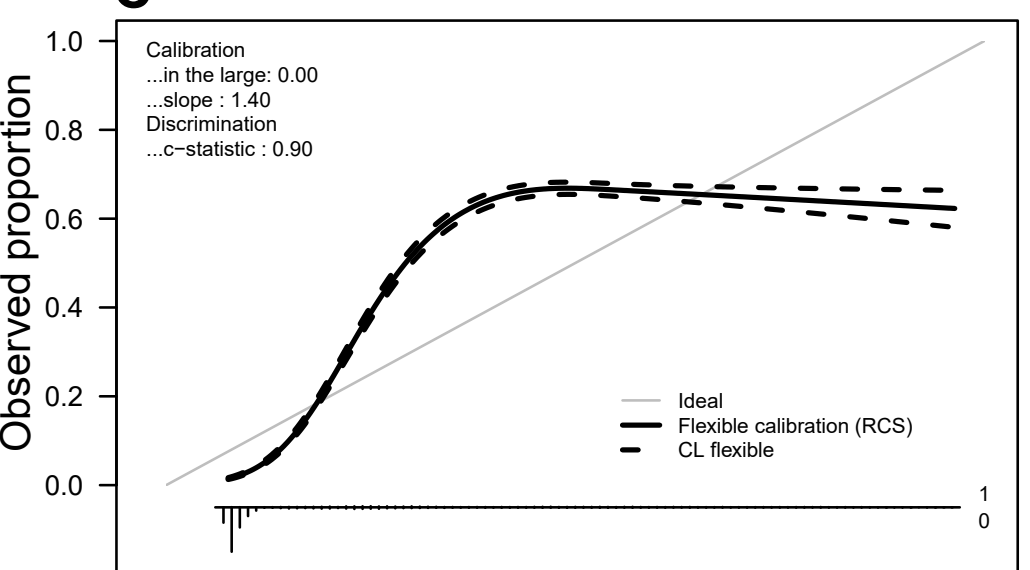

\section{D}

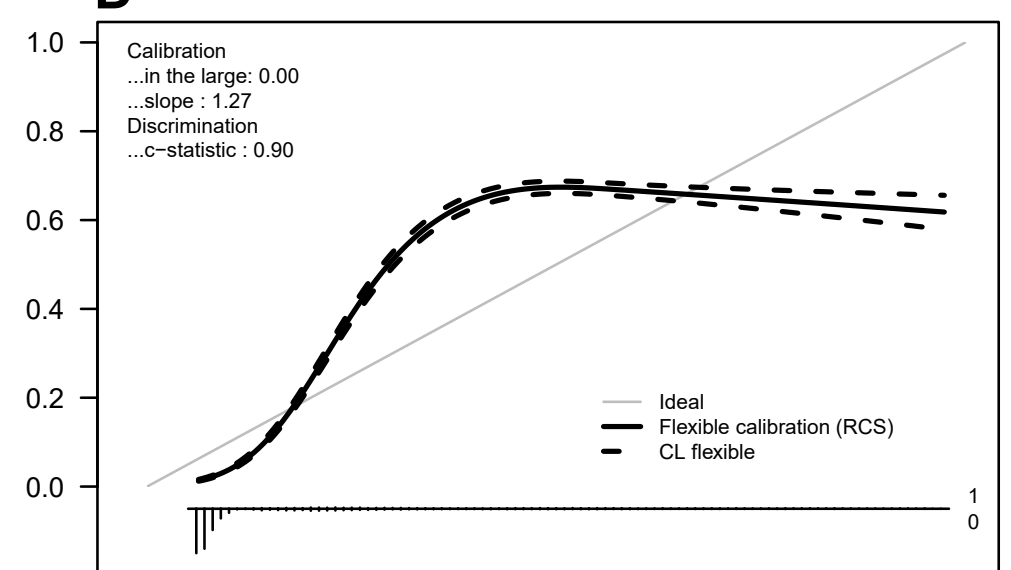

\section{E}

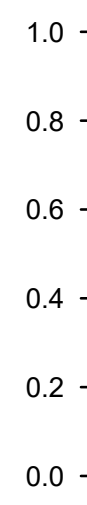

0
0
0
0
0
0
0
0
0 Calibration

...in the large: 0.00

...slope : 1.18

Discrimination
c-statistic : 0.90

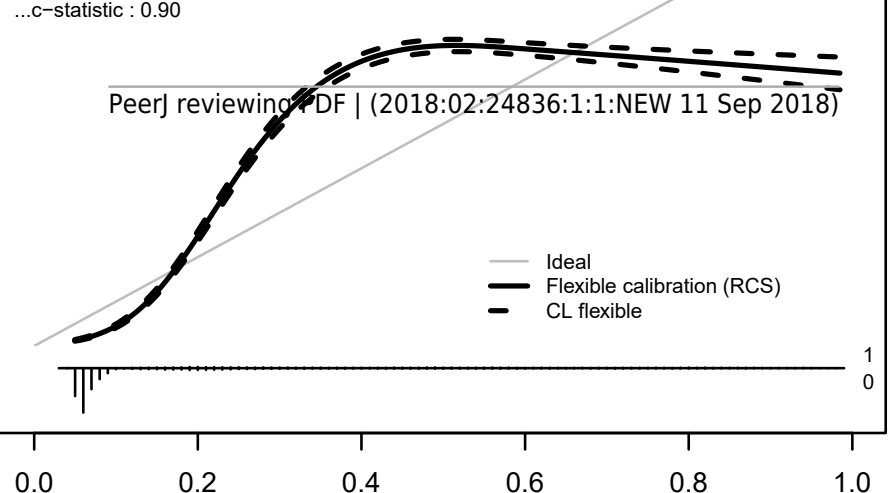

F

MND=10

Calibration

...in the large: 0.00

...slope : 1.90

Manuscrgot to be reviewed

- Flexible calibration (RCS)

- CL flexible

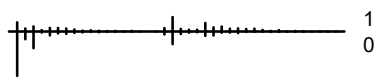

G

$M N D=20$

Calibration
...in the large: 0.00

...slope : 1.68

...c-statistic : 0.78
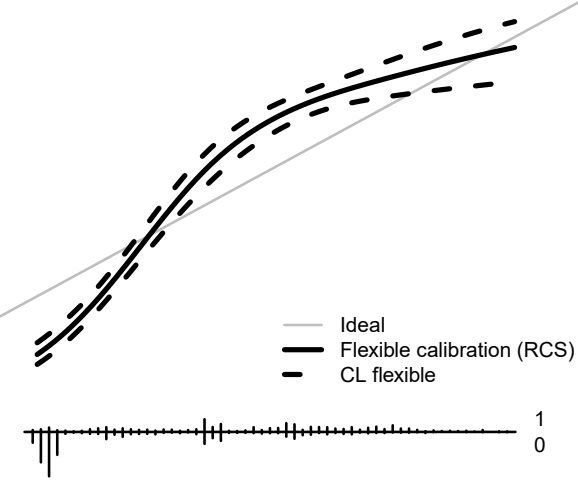

\section{$\mathrm{H} \quad \mathrm{MND}=50$}
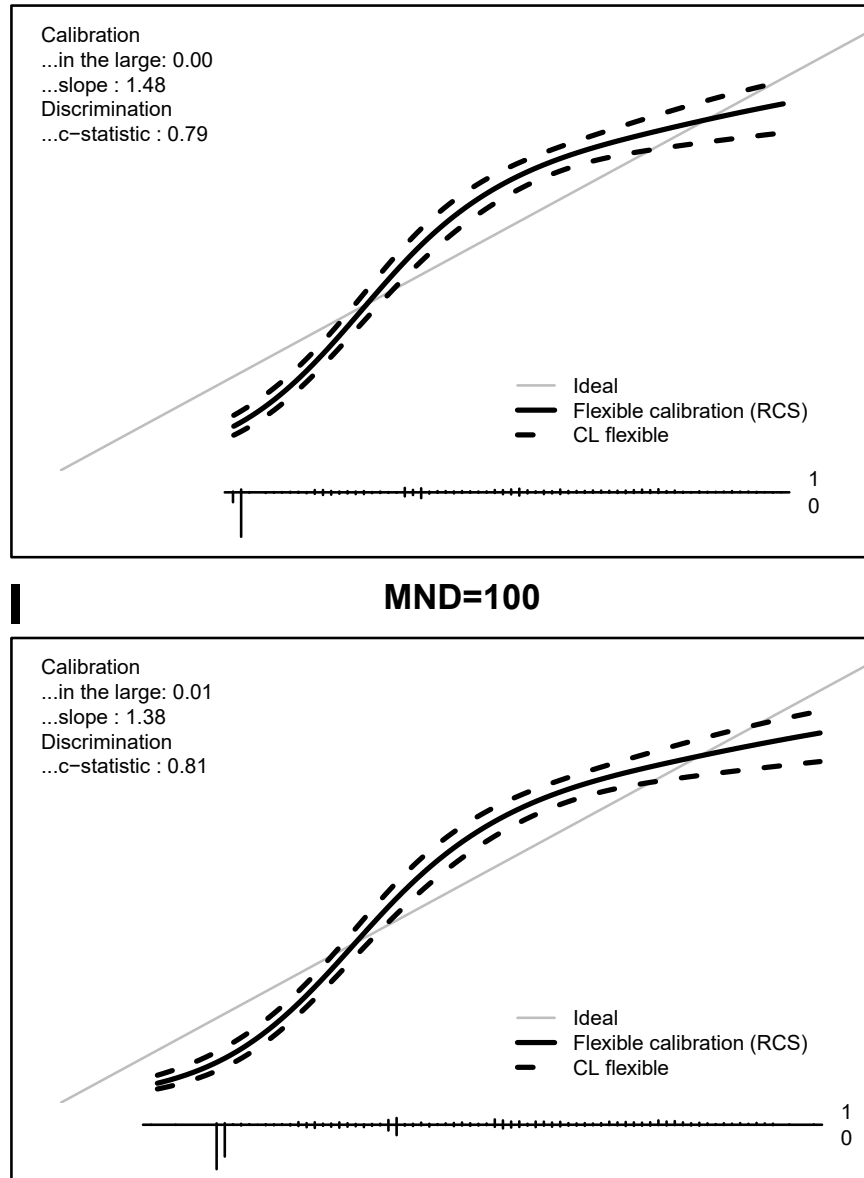

\section{J}

$M N D=N D R$

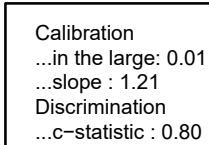

\section{.}


Table $\mathbf{1}$ (on next page)

Selected ATC codes for CVD and T2D. 
1

\begin{tabular}{|l|l|l|}
\hline Condition & ATC & Description \\
\hline T2D & A10 & Drugs used in diabetes. \\
\hline CVD & B01AA, B01AC & Cardiac agents (excl. ACE inhibators). \\
\cline { 2 - 3 } & C01, C04A & Antihypertensives. \\
\cline { 2 - 3 } & C02, C07 & Peripheral vasodilators.. \\
\cline { 2 - 3 } & C08, C09 & E.g., Beta blocking agents, Calcium channel blockers. \\
\hline
\end{tabular}

2 
Table 2 (on next page)

Summary table for predictor variables. 
1

\begin{tabular}{|l|l|l|l|l|}
\hline Variable & \multicolumn{2}{|c|}{ T2D } & \multicolumn{2}{c|}{ CVD } \\
\hline & Pos (n=934) & Neg (n=1,147) & Pos (n=3,464) & Neg (n=12,495) \\
\hline $\begin{array}{l}\text { Age } \\
\text { [95\% Cl years)] }\end{array}$ & $\begin{array}{l}66.51 \\
{[66.03-67.00]}\end{array}$ & $\begin{array}{l}65.34 \\
64.91-65.76]\end{array}$ & $\begin{array}{l}67.69 \\
{[67.42-67.99]}\end{array}$ & $\begin{array}{l}65.72 \\
{[65.59-65.86]}\end{array}$ \\
\hline Female [n (\%)] & $393(42 \%)$ & $437(38 \%)$ & $1854(54 \%)$ & $5761(46 \%)$ \\
\hline Male [n (\%)] & $541(58 \%)$ & $710(62 \%)$ & $1610(46 \%)$ & $6734(54 \%)$ \\
\hline Hosp [n (\%)] & $125(13 \%)$ & $137(12 \%)$ & $646(19 \%)$ & $1484(12 \%)$ \\
\hline \#ATC [m (\%)] & $246(36 \%)$ & $179(26 \%)$ & $352(29 \%)$ & $317(26 \%)$ \\
\hline \#ATC3 [m (\%)] & $51(8 \%)$ & $43(6 \%)$ & $59(5 \%)$ & $56(5 \%)$ \\
\hline \#ICD [m (\%)] & $234(35 \%)$ & $234(35 \%)$ & $510(42 \%)$ & $674(55 \%)$ \\
\hline
\end{tabular}

2 


\section{Table 3 (on next page)}

Number of all and stable selected variables in all experimental repetitions. Number of stable variables is presented in brackets. 
1

\begin{tabular}{|l|l|l|l|l|l|l|l|l|l|l|l|}
\hline MND & $\mathbf{1 0}$ & $\mathbf{2 0}$ & $\mathbf{3 0}$ & $\mathbf{4 0}$ & $\mathbf{5 0}$ & $\mathbf{6 0}$ & $\mathbf{7 0}$ & $\mathbf{8 0}$ & $\mathbf{9 0}$ & $\mathbf{1 0 0}$ & NDR \\
\hline CVD & $9(4)$ & $\begin{array}{l}21 \\
(15)\end{array}$ & $\begin{array}{l}32 \\
(17)\end{array}$ & $\begin{array}{l}47 \\
(25)\end{array}$ & $\begin{array}{l}66 \\
(29)\end{array}$ & $\begin{array}{l}75 \\
(30)\end{array}$ & $\begin{array}{l}89 \\
(31)\end{array}$ & $\begin{array}{l}107 \\
(32)\end{array}$ & $\begin{array}{l}119 \\
(32)\end{array}$ & $\begin{array}{l}125 \\
(33)\end{array}$ & $\begin{array}{l}389 \\
(43)\end{array}$ \\
\hline T2D & $\begin{array}{l}11 \\
(4)\end{array}$ & $\begin{array}{l}21 \\
(12)\end{array}$ & $\begin{array}{l}37 \\
(14)\end{array}$ & $\begin{array}{l}52 \\
(14)\end{array}$ & $\begin{array}{l}71 \\
(14)\end{array}$ & $\begin{array}{l}81 \\
(14)\end{array}$ & $\begin{array}{l}91 \\
(15)\end{array}$ & $\begin{array}{l}102 \\
(15)\end{array}$ & $\begin{array}{l}126 \\
(17)\end{array}$ & $\begin{array}{l}146 \\
(18)\end{array}$ & $\begin{array}{l}352 \\
(23)\end{array}$ \\
\hline
\end{tabular}

2 


\section{Table 4 (on next page)}

Ratio of all and stable selected variables in all experimental repetitions. Ratio of stable variables is presented in brackets. 
1

\begin{tabular}{|l|l|l|l|l|l|l|l|l|l|l|l|}
\hline MND & $\mathbf{1 0}$ & $\mathbf{2 0}$ & $\mathbf{3 0}$ & $\mathbf{4 0}$ & $\mathbf{5 0}$ & $\mathbf{6 0}$ & $\mathbf{7 0}$ & $\mathbf{8 0}$ & $\mathbf{9 0}$ & $\mathbf{1 0 0}$ & NDR \\
\hline CVD & $\begin{array}{l}0.03 \\
(0.09)\end{array}$ & $\begin{array}{l}0.05 \\
(0.35)\end{array}$ & $\begin{array}{l}0.08 \\
(0.4)\end{array}$ & $\begin{array}{l}0.10 \\
(0.58)\end{array}$ & $\begin{array}{l}0.13 \\
(0.67)\end{array}$ & $\begin{array}{l}0.15 \\
(0.7)\end{array}$ & $\begin{array}{l}0.18 \\
(0.72)\end{array}$ & $\begin{array}{l}0.21 \\
(0.74)\end{array}$ & $\begin{array}{l}0.23 \\
(0.74)\end{array}$ & $\begin{array}{l}0.26 \\
(0.77)\end{array}$ & $\begin{array}{l}1.00 \\
(1.00)\end{array}$ \\
\hline T2D & $\begin{array}{l}0.01 \\
(0.17)\end{array}$ & $\begin{array}{l}0.01 \\
(0.52)\end{array}$ & $\begin{array}{l}0.02 \\
(0.61)\end{array}$ & $\begin{array}{l}0.03 \\
(0.61)\end{array}$ & $\begin{array}{l}0.04 \\
(0.61)\end{array}$ & $\begin{array}{l}0.05 \\
(0.61)\end{array}$ & $\begin{array}{l}0.06 \\
(0.65)\end{array}$ & $\begin{array}{l}0.07 \\
(0.65)\end{array}$ & $\begin{array}{l}0.08 \\
(0.74)\end{array}$ & $\begin{array}{l}0.08 \\
(0.78)\end{array}$ & $\begin{array}{l}1.00 \\
(1.00)\end{array}$ \\
\hline
\end{tabular}

2 